\title{
Variable range hopping and thermally activated transport in molybdenum-based MXenes
}

\author{
Joseph Halim, ${ }^{1}$ Eun Ju Moon, ${ }^{2}$ Per Eklund, ${ }^{1}$ Johanna Rosen, ${ }^{1}$ Michel W. Barsoum, ${ }^{2, *}$ and Thierry Ouisse ${ }^{3, \dagger}$ \\ ${ }^{1}$ Thin Film Physics Division, Department of Physics, Chemistry and Biology (IFM), Linköping University, SE-581 83 Linköping, Sweden \\ ${ }^{2}$ Department of Materials Science and Engineering, Drexel University, Philadelphia, Pennsylvania 19104, USA \\ ${ }^{3}$ Université Grenoble-Alpes, Centre National de la Recherche Scientifique, LMGP, F-38000 Grenoble, France
}

(Received 5 June 2018; revised manuscript received 7 August 2018; published 10 September 2018)

\begin{abstract}
The magnetotransport of freestanding, vacuum filtered, thin films of $\mathrm{Mo}_{2} \mathrm{CT}_{z}, \mathrm{Mo}_{1.33} \mathrm{CT}_{z}, \mathrm{Mo}_{2} \mathrm{TiC}_{2} \mathrm{~T}_{z}$, and $\mathrm{Mo}_{2} \mathrm{Ti}_{2} \mathrm{C}_{3} \mathrm{~T}_{z}$ was measured in the $10-300-\mathrm{K}$ temperature $(T)$ range. Some of the films were annealed before measuring their transport properties. Analysis of the results suggest that-with the exception of the heavily defective $\mathrm{Mo}_{1.33} \mathrm{CT}_{z}$ composition-in the 10- to 200-K temperature regime, variable range hopping between individual MXene sheets is the operative conduction mechanism. For $\mathrm{Mo}_{1.33} \mathrm{CT}_{z}$ it is more likely that variable range hopping within individual flakes is rate limiting. At higher temperatures, a thermally activated process emerges in all cases. It follows that improved fabrication processes should lead to considerable improvements in the electrical transport of Mo-based MXenes.
\end{abstract}

DOI: 10.1103/PhysRevB.98.104202

\section{INTRODUCTION}

MXenes, a recently discovered family of two-dimensional (2D) carbides and nitrides [1,2], are obtained and so called because they are synthesized by chemically etching the A element (mainly groups 13 and 14) from their parent MAX phases. The latter are layered, ternary, early transition metal, $\mathrm{M}$, carbides and/or nitrides with a stoichiometry $\mathrm{M}_{n+1} \mathrm{AX}_{n}$, where $\mathrm{X}$ is either $\mathrm{C}$ or $\mathrm{N}$ and $\mathrm{n}$ varies between 1 and 3 [3]. With a few exceptions, such as $\mathrm{Mo}_{2} \mathrm{Ga}_{2} \mathrm{C}$ (where the Ga layers are etched) [4], most other MXenes have been obtained by etching the $\mathrm{Al}$ layers from Al-containing MAX phases using, among other etchants, hydrofluoric acid (HF) or a combination of a $\mathrm{LiF}$ salt and $\mathrm{HCl}[5,6]$. When the A layers are etched, they are replaced by surface terminations, $\mathrm{T}$ $(-\mathrm{O},-\mathrm{OH}$, and/or $-\mathrm{F})$, which is why their proper designation is $\mathrm{M}_{n+1} \mathrm{X}_{n} \mathrm{~T}_{z}$ [7]. MXenes stand out in several ways: they exhibit good electrical conductivities [8], are hydrophilic [2], are chemically tailorable $[9,10]$, and possess - at the Fermi level $\mathrm{E}_{\mathrm{F}}-\mathrm{a}$ high density of states, $\mathrm{D}\left(\mathrm{E}_{\mathrm{F}}\right)$ [11-13]. Partially because of these properties, MXenes have shown promise for use in supercapacitors [14], electromagnetic shielding $[15,16]$, sensors [17-20], electrode materials for $\mathrm{Li}$ and multivalent ion batteries [21], electrochemical catalytic surfaces [22], hydrogen storage [23], thermoelectric materials [24], and transparent 2D conductors $[25,26]$.

Currently, the majority of the research on MXenes has focused on the $\mathrm{Ti}_{3} \mathrm{C}_{2} \mathrm{~T}_{z}$-based composition as it was the first to be discovered. However, recently there has been considerable interest in investigating Mo-based MXenes. These compounds have shown promise in several applications such as energy storage [10,26-29], hydrogen production [30], thermoelectrics [24], and water desalination [31] among others.

\footnotetext{
*Corresponding author: barsoumw@drexel.edu

${ }^{\dagger}$ Corresponding author: thierry.ouisse@grenoble-inp.fr
}

To date, four Mo-based MXenes, viz., $\mathrm{Mo}_{2} \mathrm{TiC}_{2} \mathrm{~T}_{z}$, $\mathrm{Mo}_{2} \mathrm{Ti}_{2} \mathrm{C}_{3} \mathrm{~T}_{z}, \mathrm{Mo}_{2} \mathrm{CT}_{z}$, and $\mathrm{Mo}_{1.33} \mathrm{CT}_{z}$, have been discovered $[10,28,32]$. The first two are synthesized by etching the Al layers from the out-of-plane ordered MAX phases: $\mathrm{Mo}_{2} \mathrm{TiAlC}_{2}$ and $\mathrm{Mo}_{2} \mathrm{Ti}_{2} \mathrm{AlC}_{3}[33,34]$. In these MAX phases, the layers bonded to the $\mathrm{Al}$ layers are mostly composed of Mo; the inner layers are mostly Ti. When these phases are etched, the $\mathrm{Al}$ layers are replaced by surface termination groups, while the Ti layers are situated in the inner layers that are bonded to $\mathrm{C}$ atoms only. The $\mathrm{Mo}_{2} \mathrm{CT}_{z}$ is synthesized by etching the Ga layers from a MAX-like phase, $\mathrm{Mo}_{2} \mathrm{Ga}_{2} \mathrm{C}$ [4]. The $\mathrm{Mo}_{1.33} \mathrm{CT}_{z}$ contains ordered vacancies created when the $\mathrm{Sc}$ and $\mathrm{Al}$ atoms are etched from the in-plane ordered i-MAX phase $\left(\mathrm{Mo}_{2 / 3} \mathrm{Sc}_{1 / 3}\right)_{2} \mathrm{AlC}$, where Mo and $\mathrm{Sc}$ are ordered in a $2: 1$ ratio in the basal plane [28].

MXenes films are often processed by vacuum filtering aqueous colloidal suspensions of delaminated flakes through polymer nanoporous filters. In most cases the resulting thin films are freestanding and flexible [32]. Figure 1 shows a typical scanning electron microscope (SEM) micrograph of such a film. Also see SEM images in Fig. S1 in Sec. I of the Supplemental Material [35] and Refs. [24,32].

In many MXene applications, such as in energy storage devices, transparent conductive electrodes, and catalysis, the performance depends critically on the electronic properties of a collection of flakes. Understanding the microscopic mechanisms governing the electrical conductivities of MXenes is therefore key, both for their intrinsic academic merit and for applications. The conductivity of the first discovered and most understood MXene to date, $\mathrm{Ti}_{3} \mathrm{C}_{2} \mathrm{~T}_{z}$, is for the most part metalliclike, in that the resistance decreases linearly with decreasing temperatures in the 50-300-K temperature range. Below $50 \mathrm{~K}$, the resistance has a tendency to increase slightly with decreasing temperatures $[8,26,36]$.

This paper deals with the transport mechanisms of Mobased MXenes. In general, compared to $\mathrm{Ti}_{3} \mathrm{C}_{2} \mathrm{~T}_{z}$, Mo-based MXenes are more resistive (see, e.g., [24,32]). The lowest 


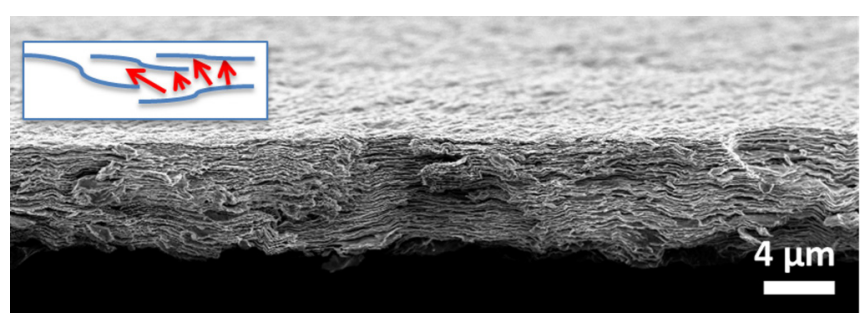

FIG. 1. Cross-sectional SEM micrograph of $\mathrm{Mo}_{2} \mathrm{CT}_{z}$ unannealed freestanding film. Inset of a schematic showing the interflake transport mechanism.

room-temperature (RT) resistivity, $\rho$, values obtained to date are of the order of $6.6 \mu \Omega \mathrm{m}$ [37]; for $\mathrm{Ti}_{3} \mathrm{C}_{2} \mathrm{~T}_{x}$ films, the corresponding value is $1.3 \mu \Omega \mathrm{m}[38,39]$. Another important distinction between $\mathrm{Ti}_{3} \mathrm{C}_{2} \mathrm{~T}_{z}$ and Mo-based MXenes is the fact that for the latter $\rho$ increases with decreasing temperatures [24,32]. This is somewhat puzzling since density functional theory (DFT) predicts that most MXenes should possess a high density of states at the Fermi level, $\mathrm{D}\left(\mathrm{E}_{\mathrm{F}}\right)$ [11]. For example, recently Lind et al. [40] used DFT calculations to shed light on the electronic properties of $\mathrm{Mo}_{2} \mathrm{CT}_{z}$ and $\mathrm{Mo}_{1.33} \mathrm{CT}_{z}$. In both cases, $\mathrm{D}\left(\mathrm{E}_{\mathrm{F}}\right)$ was a function of termination chemistries. For example, $\mathrm{D}\left(\mathrm{E}_{\mathrm{F}}\right)$ for $\mathrm{Mo}_{1.33} \mathrm{CF}_{2}$ and $\mathrm{Mo}_{1.33} \mathrm{C}\left(\mathrm{O}_{0.33}, \mathrm{~F}_{0.67}\right)_{2}$ were predicted to be, respectively, 0.65 and $4.32 \mathrm{eV}^{-1}$ states per formula unit, where the latter is composed of four Mo, three $\mathrm{C}$, and six termination atoms, viz., $\mathrm{Mo}_{4} \mathrm{C}_{3} \mathrm{O}_{2} \mathrm{~F}_{4}$. The same is true of $\mathrm{Mo}_{2} \mathrm{TiC}_{2} \mathrm{~T}_{z}$ and $\mathrm{Mo}_{2} \mathrm{Ti}_{2} \mathrm{C}_{3} \mathrm{~T}_{z}$ [37].

The fundamental question this paper tries to shed light on is the following: Why, if $\mathrm{D}\left(\mathrm{E}_{\mathrm{F}}\right)$ is so high, does $\rho$ increase with decreasing temperature? This is especially germane since most papers relating to the transport of Mo-based MXene describe the transport as semiconductorlike, with the implication that a gap of sorts may be opening up $[24,32,37,40]$. To answer this question, we measured the temperature variation of magnetotransport in Mo-based MXenes in order to understand the nature of electron transport and microscopic processes at play. This is achieved through analysis of transport data measured herein and, previously, on Mo-based MXene freestanding thin films with various stoichiometries and processing conditions. As shown below, transport in most compositions can be described by a variable range hopping (VRH) mechanism at lower temperatures supplemented by a parallel, thermally activated, mechanism at higher temperatures. In the VRH mechanism, the charge carriers are assumed to hop from flake to flake across a distribution of distances as shown schematically in the inset in Fig. 1. Most of our compositions share characteristic features already observed in systems where highly conductive entities are separated by thin disordered regions such as granular metals and cermets [41-50]. The latter are defined herein as composites with high volume fractions of metal particles separated from each other by thin insulating layers.

\section{EXPERIMENTAL DETAILS}

\section{A. Materials synthesis}

The delaminated freestanding $\mathrm{Mo}_{2} \mathrm{TiC}_{2} \mathrm{~T}_{z}$ and $\mathrm{Mo}_{2} \mathrm{Ti}_{2} \mathrm{C}_{3} \mathrm{~T}_{z}$ films used herein were cut from the same larger film of the same compounds used in Ref. [37]. In other words, transport properties of different regions of the same films were measured. This was carried out to explore the reproducibility of the films tested at different times by different researchers. In short, both films were made by first adding $2 \mathrm{~g}$ of $\mathrm{Mo}_{2} \mathrm{TiAlC}_{2}$ or $\mathrm{Mo}_{2} \mathrm{Ti}_{2} \mathrm{AlC}_{3}$ powders [33,34] to $20 \mathrm{ml}$ of $50 \%$ aqueous $\mathrm{HF}$ solution. The mixtures were held at $55^{\circ} \mathrm{C}$ for 48 and $90 \mathrm{~h}$, respectively. After etching, the mixtures were washed with deionized (DI) water till the supernatant reached a $\mathrm{pH}$ of $\approx 6$. The solid content was separated from its liquid product by filtering through a nanoporous polypropylene membrane (3501 coated PP, $0.064-\mu \mathrm{m}$ pore size, Celgard, USA). At this stage the films break down into powders.

One gram of each of the resulting powders was then mixed with $10 \mathrm{ml}$ of 40-wt.-\% tetrabutylammonium hydroxide (TBAOH) and stirred for $4 \mathrm{~h}$ at RT. The resulting mixture was centrifuged, and the supernatant was decanted and discarded. $50 \mathrm{ml}$ of DI water was added to the residue and centrifuged to remove any residual TBAOH. After decanting the supernatant, $100 \mathrm{ml}$ of DI water was added to the residue and the mixture was sonicated for $1 \mathrm{~h}$, then centrifuged for $1 \mathrm{~h}$ at $5000 \mathrm{rpm}$. Freestanding thin films of both compounds were produced by filtering the supernatant containing delaminated MXene flakes through the nanoporous polypropylene membrane. As discussed below, the reproducibility was excellent, and thus instead of carrying our own annealing experiments on these two compositions, we simply discuss the results published previously [37].

Delaminated freestanding thin films of $\mathrm{Mo}_{2} \mathrm{CT}_{z}$ were produced by adding $2 \mathrm{~g}$ of $\mathrm{Mo}_{2} \mathrm{Ga}_{2} \mathrm{C}$ powder [32] in $80 \mathrm{ml}$ of a 25-wt.-\% HF aqueous solution. The mixture was held at $55^{\circ} \mathrm{C}$ for $144 \mathrm{~h}$ then washed with DI water till a $\mathrm{pH}>6$ was reached. After washing, the MXene dispersion was centrifuged and the supernatant was discarded. Then, $5 \mathrm{ml}$ of $40 \%$ TBAOH was mixed with $15 \mathrm{ml} \mathrm{DI}$ water and half that mixture $(10 \mathrm{ml})$ was added to $1 \mathrm{~g}$ of the etched $\mathrm{Mo}_{2} \mathrm{CT}_{z}$ powder. The mixture was shaken using a vortex mixer for $5 \mathrm{~min}$, then allowed to sit for $10 \mathrm{~min}$. Afterwards, the mixture was centrifuged at $5000 \mathrm{rpm}$ for $10 \mathrm{~min}$, followed by decanting the supernatant. DI water $(45 \mathrm{ml})$ was then added and the mixture was centrifuged at $5000 \mathrm{rpm}$ for $4 \mathrm{~min}$ and again decanting the supernatant. This was repeated three times.

In order to ensure the removal of any $\mathrm{TBAOH}$ residue, $45 \mathrm{ml}$ of 200 proof ethanol was added to the residue and blended for $2 \mathrm{~min}$ in a vortex mixer and centrifuged at 5000 $\mathrm{rpm}$ for $10 \mathrm{~min}$. This process was repeated twice. Then, 15 $\mathrm{ml}$ of DI water was added to the residue and the mixture was shaken using the vortex mixer for $7 \mathrm{~min}$ and centrifuged for $1 \mathrm{~h}$ at $5000 \mathrm{rpm}$. Lastly, about $15 \mathrm{ml}$ of the supernatant was filtered through the Celgard membrane to fabricate a $\mathrm{Mo}_{2} \mathrm{CT}_{z}$ thin film composed of restacked delaminated thin flakes. This film was easily peeled off the membrane, but its initial $\rho$ was quite high. To decrease its $\rho$, the sample was annealed in vacuum at $200{ }^{\circ} \mathrm{C}$ for $32 \mathrm{~h}$ before testing.

The $\mathrm{Mo}_{1.33} \mathrm{CT}_{z}$ delaminated freestanding film was produced by first adding $1 \mathrm{~g}$ of $\left(\mathrm{Mo}_{2 / 3} \mathrm{Sc}_{1 / 3}\right)_{2} \mathrm{AlC}$ to $20 \mathrm{ml} \mathrm{48-}$ wt.-\% HF. The mixture was set for $24 \mathrm{~h}$ at RT as in Ref. [28]. After etching, the MXene multilayers were washed with DI water till a $\mathrm{pH}>6$ was reached. After filtering and drying, 
$1 \mathrm{~g}$ of powder and $10 \mathrm{ml}$ of TBAOH were mixed together and manually shaken for $5 \mathrm{~min}$ followed by centrifuging at 6000 rpm for $5 \mathrm{~min}$. The supernatant was discarded and $45 \mathrm{ml}$ of DI water was added to the residue to get rid of any TBAOH leftover. The mixture was centrifuged at $5000 \mathrm{rpm}$ for $1 \mathrm{~min}$ and the supernatant was decanted; this process was repeated three times. To delaminate the MXene, $45 \mathrm{ml}$ of DI water was mixed with the MXene treated with TBAOH and manually shaken for $5 \mathrm{~min}$ and centrifuged at $5000 \mathrm{rpm}$ for $1 \mathrm{~h}$. Lastly, about $15 \mathrm{ml}$ of the colloidal suspension was filtered through the polymer membrane to fabricate a thin film composed of restacked $\mathrm{Mo}_{1.33} \mathrm{C}$ delaminated flakes.

\section{B. Characterization techniques}

A SEM (Zeiss Surpa 50 VP, Germany) was used for image cross sections of filtered thin $\mathrm{Mo}_{2} \mathrm{CT}_{z}$ and $\mathrm{Mo}_{1.33} \mathrm{CT}_{z}$ films. X-ray-diffraction (XRD) patterns were collected using a diffractometer (Bruker, D8 advance, AXS system, Germany) with $\mathrm{Cu} \mathrm{K} \alpha$ radiation; $\lambda=1.5406 \AA$. Note that the main peak at the lowest angles is half the $c$-lattice parameter $(c$-LP) or (002) peaks - based on the symmetry of the parent MAX phase-and will henceforth be referred to as the interlayer distance or $\mathrm{d}_{\mathrm{c} / 2}$ interchangeably.

Resistivity $(\rho)$ versus temperature $(T)$, magnetoresistance $(\mathrm{MR})$, and Hall voltage $\left(V_{\mathrm{H}}\right)$ measurements were carried out in a Quantum Design Physical Property Measurement System, equipped with an external Keithley current source and a nanovoltmeter. Electrical contact was made by attaching silver wires in a Van der Pauw configuration to the films using silver paint (Structure Probe, Inc., PA, USA). The MR and $V_{\mathrm{H}}$ were measured as the magnetic field was cycled between $\pm 8 \mathrm{~T}$, applied out of the plane of the samples. The measured $V_{\mathrm{H}}$ contained contributions from $\rho$ and MR. To remove the latter, a parabolic component was first subtracted out and the resulting voltage was symmetrized for negative and positive magnetic fields similar to the procedure outlined in Ref. [51].

\section{RESULTS AND DISCUSSION}

Typical low-angle (0002 peaks) XRD patterns of $\mathrm{Mo}_{2} \mathrm{CT}_{z}$ before and after annealing are plotted in Fig. 2. The $\mathrm{d}_{\mathrm{c} / 2}$ value, or interlayer spacing, is denoted in the figure by vertical dashed lines. From these results, it is clear that (i) the peaks are quite broad and not very well defined and (ii) annealing shifts the centroid of the peaks towards larger $2 \theta$ values, viz., smaller $\mathrm{d}_{\mathrm{c} / 2}$ spacings.

Figure 3 shows the temperature dependencies of $\rho$ obtained herein and in previous work. Figures 4(a) and 4(b) are plots of, respectively, the dependencies of the conductivities, $\sigma$, on $\mathrm{d}_{\mathrm{c} / 2}$ and on annealing temperatures. Table I summarizes the $\mathrm{d}_{\mathrm{c} / 2}$ and RT conductivity, $\sigma_{\mathrm{RT}}$, values of the same set of results. Also listed in Table I are the parent phases and their $\mathrm{d}_{\mathrm{c} / 2}$ values.

Before analyzing our results, it is important to point out some general trends that any attempts to model these materials need to contend with. There are essentially four chemistries: $\mathrm{Mo}_{2} \mathrm{CT}_{z}, \mathrm{Mo}_{1.33} \mathrm{CT}_{z}, \mathrm{Mo}_{2} \mathrm{TiC}_{2} \mathrm{~T}_{z}$, and $\mathrm{Mo}_{2} \mathrm{Ti}_{2} \mathrm{C}_{3} \mathrm{~T}_{z}$. With only two exceptions, labelled multilayers Table I in which multilayered cold pressed disks were tested (added for the

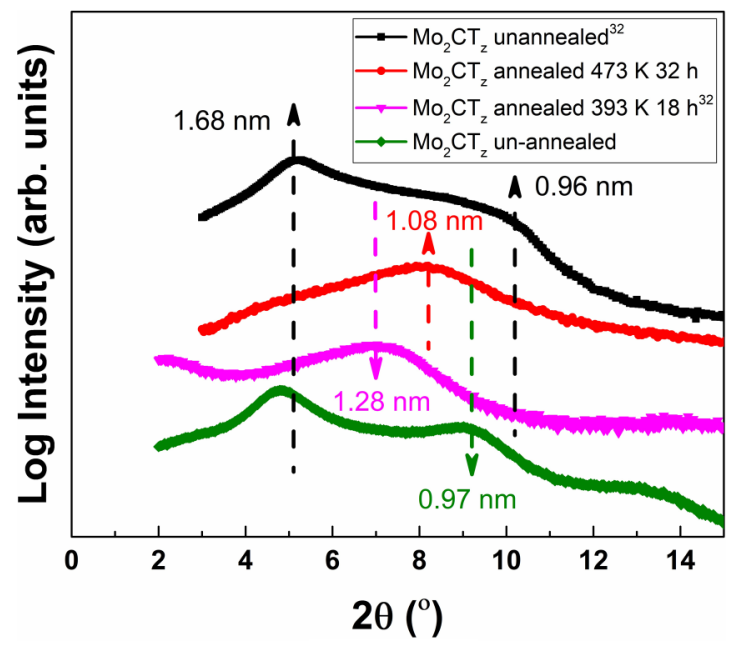

FIG. 2. XRD patterns for $\mathrm{Mo}_{2} \mathrm{CT}_{z}$ freestanding films, showing the (0002) peaks. The black curve is for unannealed film and the purple curve is for annealed film at $393 \mathrm{~K}$ for $18 \mathrm{~h}$, both obtained from Ref. [32]; the red curve is for annealed film at $473 \mathrm{~K}$ for $32 \mathrm{~h}$; and the green curve is for unannealed thin film for this paper. The $d_{c / 2}$ values, or interlayer spacings, are denoted in the figure by vertical dashed lines.

sake of completion), all other results were obtained on filtered films. As noted above, in some cases, the films were annealed to enhance their conductivities. From a perusal of the results shown in Figs. 3, 4(a), and 4(b) and Table I, we have the following.

(i) Over the entire set of results, $\rho$ varies over six orders of magnitude, and in all cases increases with decreasing temperatures (Fig. 3).

(ii) The $\mathrm{Mo}_{2} \mathrm{CT}_{z}$ chemistry is the least conductive followed by $\mathrm{Mo}_{1.33} \mathrm{CT}_{z}$. The conductivities of $\mathrm{Mo}_{2} \mathrm{Ti}_{2} \mathrm{C}_{3} \mathrm{~T}_{z}$ and $\mathrm{Mo}_{2} \mathrm{TiC}_{2} \mathrm{~T}_{z}$ are comparable and high (Fig. 3). Note that there are four data sets: two from Ref. [37] and two measured herein. The reproducibility is so good that the two sets of results are almost indistinguishable. Further analysis was carried out only on our results.

(iii) $\sigma$ is a strong function of $\mathrm{d}_{\mathrm{c} / 2}$, especially for the $\mathrm{Mo}_{2} \mathrm{CT}_{z}$ films [Fig. 4(a)]. Larger $\mathrm{d}_{\mathrm{c} / 2}$ values result in less conductive films [Fig. 4(a)]. Note the logarithmic nature of the $y$ axis in Fig. 4(a). This implies that conduction from flake to flake must be rate limiting, at least, in this case.

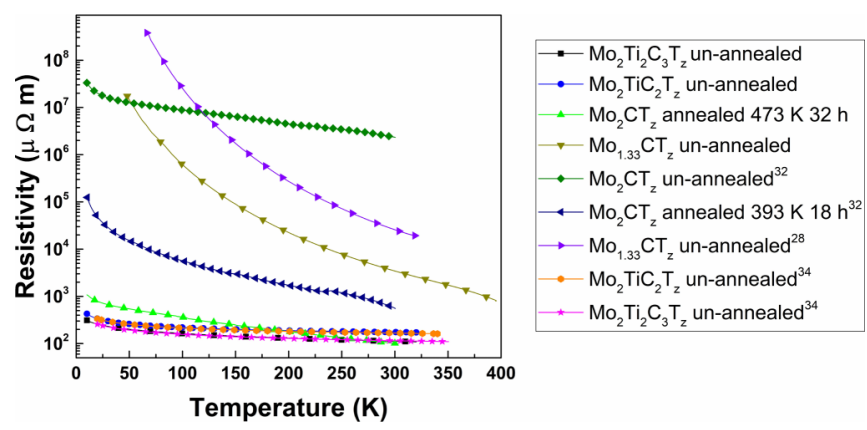

FIG. 3. Temperature dependence of resistivity for all Mo-based MXene freestanding thin films herein and those reported in the literature (see Table I). 

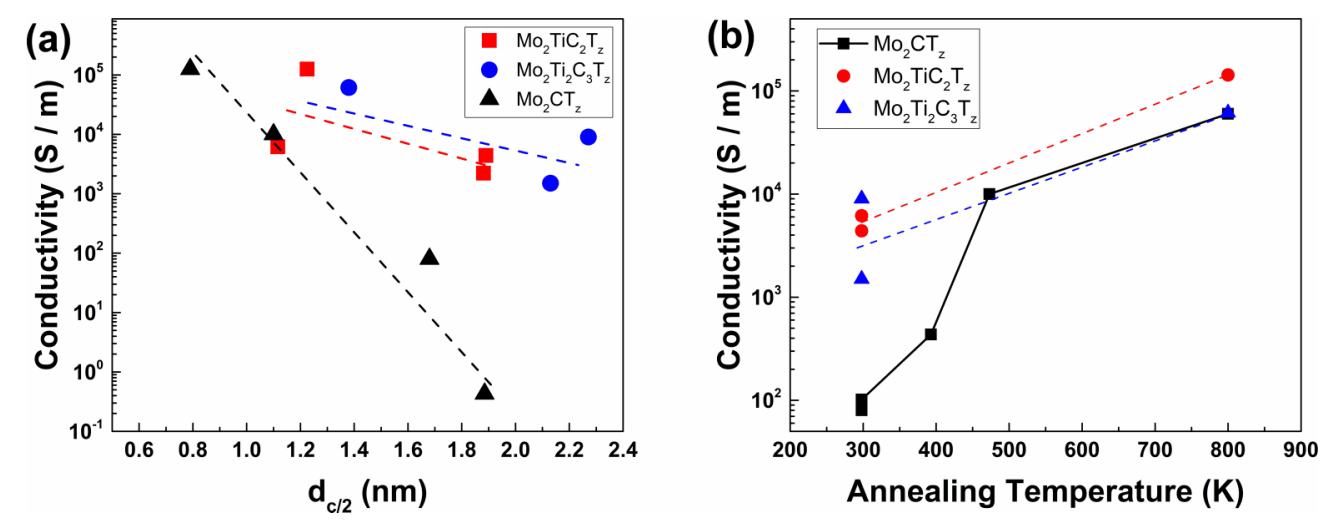

FIG. 4. Conductivity of $\mathrm{Mo}_{2} \mathrm{~T}_{2} \mathrm{C}_{3} \mathrm{~T}_{z}, \mathrm{Mo}_{2} \mathrm{TiC}_{2} \mathrm{~T}_{z}$, and $\mathrm{Mo}_{2} \mathrm{CT}_{z}$ freestanding thin films plotted against (a) interlayer distance $\mathrm{d}_{\mathrm{c} / 2}$ and (b) annealing temperatures.

(iv) Annealing temperature also has a dramatic effect on $\sigma$ [Fig. 4(b)]. Here again, the $y$ axis is logarithmic.

In the films tested herein, one can envisage two limiting regimes: one in which interflake transport is rate limiting (inset in Fig. 1) and another in which the intraflake transport is. As discussed below, there is reason to believe that transport in the highly defective $\mathrm{Mo}_{1.33} \mathrm{CT}_{z}$ films is dominated by intraflake transport. For all others, transport is, mostly, interflake limited. The latter are discussed first.

If transport were limited by the conductivities of the flakes alone (intraflake), then $\sigma$ would not be a function of $\mathrm{d}_{\mathrm{c} / 2}$. The fact that it is [Fig. 4(a)] is thus a strong indication that interflake transport is rate limiting. This is not too surprising since the flakes are terminated by insulating moieties (e.g.,
$\mathrm{O}, \mathrm{OH}$, and $\mathrm{F}$ terminations) and intercalated water molecules, and/or separated by tetrabutyl ammonium, $\mathrm{TBA}^{+}$molecules [7,52]. The fact that one of the main characteristics of MXenes is their high $\mathrm{D}\left(\mathrm{E}_{\mathrm{F}}\right)$ is consistent with this idea. Furthermore, fact that the conductivities of cold pressed discs (labeled multilayers in Table I) — composed of the same flakes as those used for the filtered films - are different from the latter is also consistent with this important conclusion. Said otherwise, the distance, orientation of the flakes relative to each other and the ir degree or overlap is important.

To shed light on the operative conduction mechanisms, we now turn to analyzing the results shown in Figs. 5 and 6 in which $\log \sigma$ is plotted versus reciprocal T. The most likely conduction mechanism applicable here is VRH [53]. In that

TABLE I. Summary of $\mathrm{d}_{\mathrm{c} / 2}$ and $\sigma_{\mathrm{RT}}$ values measured on Mo-based MXenes tested herein and those reported in the literature. Also listed are the parent phases and their lattice parameters.

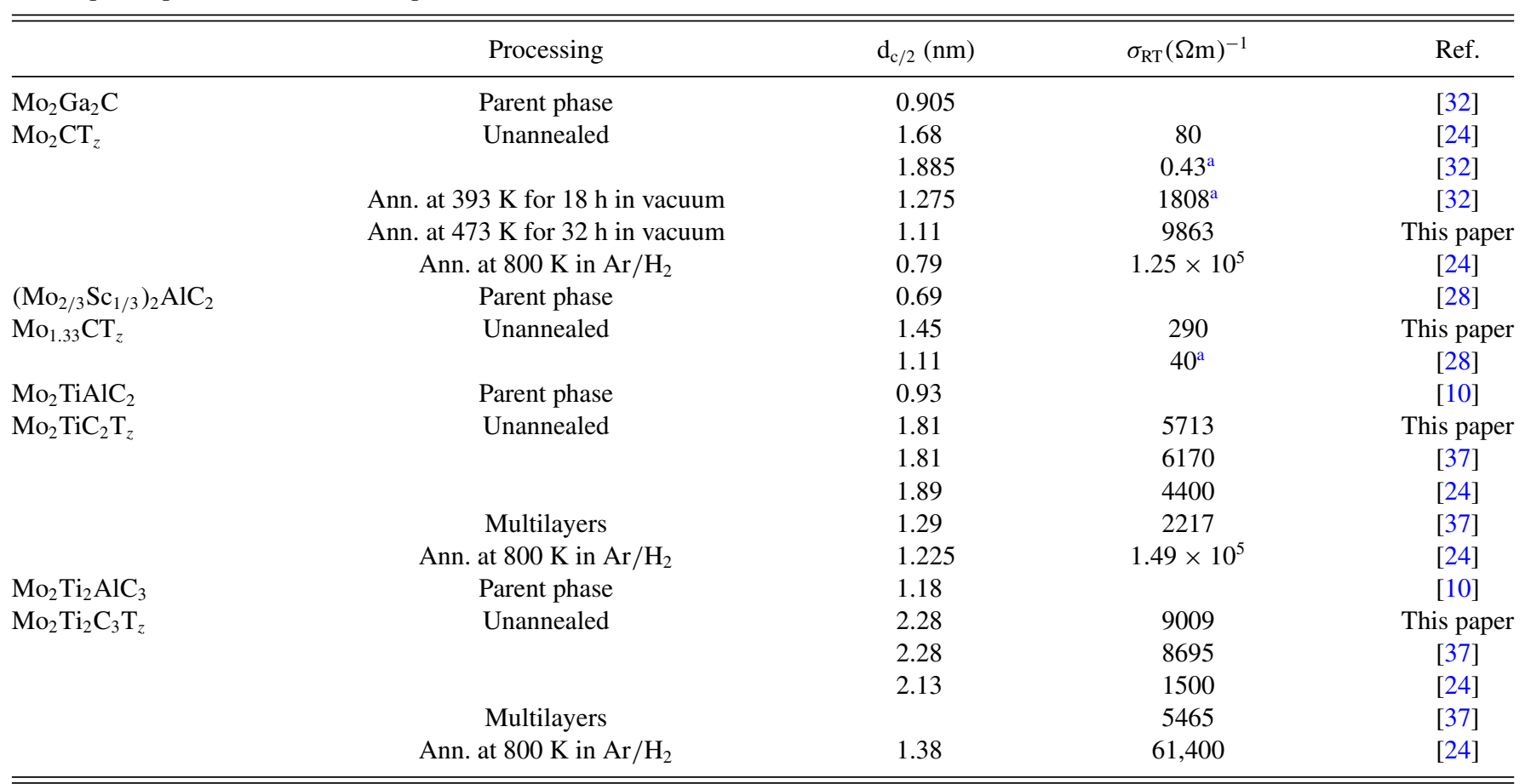

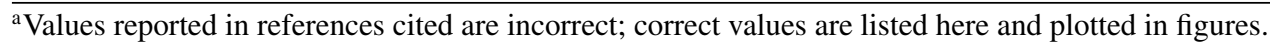



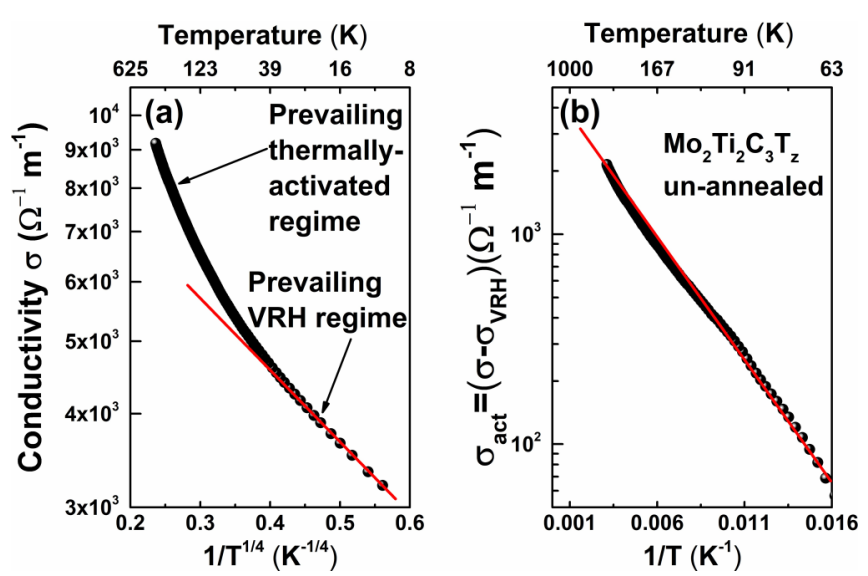

FIG. 5. Conductivity of unannealed freestanding filtered films of $\mathrm{Mo}_{2} \mathrm{Ti}_{2} \mathrm{C}_{3} \mathrm{~T}_{z}$ : (a) $\log \sigma$ vs $1 / \mathrm{T}^{1 / 4}$ (3D VRH). (b) Conductivity after subtraction of VRH component from total conductivity vs $1 / \mathrm{T}$. The resulting curve fits the thermal activation model quite well.

case, $\sigma_{\mathrm{VRH}}$ is described by

$$
\sigma_{\mathrm{VRH}}=\sigma_{0} \exp -\left(\frac{T_{0}}{T}\right)^{x}
$$

where $\sigma_{0}$ is a conductivity prefactor and $\mathrm{T}_{0}$ is a characteristic temperature with an exponent $\mathrm{x}$. The latter depends on the dimensionality of the transport, $d$, and a term $p$ characterizing the variation of $\mathrm{D}\left(\mathrm{E}_{\mathrm{F}}\right)$ with energy [54,55]. The relationship is given by

$$
x=(p+1) /(d+p+1) .
$$

If $\mathrm{D}\left(\mathrm{E}_{\mathrm{F}}\right)$ is not a strong function of energy, $\mathrm{p}=0$ and $x$ reduces to $1 / 4$ for $d=3$, and $1 / 3$ for $d=2$ [53]. In case of prominent charging or Coulomb effects (Efros-Shklovskii model [55]), $x$ is equal to $1 / 2$ and is independent of dimensionality [41].

When $\log \sigma$ of the $\mathrm{Mo}_{2} \mathrm{Ti}_{2} \mathrm{C}_{3} \mathrm{~T}_{z}$ film is plotted versus $\mathrm{T}^{1 / 4}$ [Fig. 5(a)] it is clear that the fit is excellent at lower temperatures. However, a second mechanism kicks in at higher temperatures. It follows that the low-T range is dominated by a law of the form given by Eq. (1) while another, higher, T range might be thermally activated. We thus assume that the total conductivity, $\sigma_{\text {tot }}$, is the sum of

$$
\sigma_{\mathrm{tot}}=\sigma_{\mathrm{VRH}}+\sigma_{\mathrm{act}}
$$

where $\sigma_{\text {act }}$ is due to a thermally activated process. When $\log \sigma_{\text {act }}=\sigma_{\text {tot }}-\sigma_{\mathrm{VRH}}$ is plotted versus $1 / \mathrm{T}[$ Fig. $5(\mathrm{~b})]$ it is indicated that $\sigma_{\text {act }}$ is indeed thermally activated with an equation of the form

$$
\sigma_{\mathrm{act}}=\sigma_{A} \exp -\frac{T_{A}}{T}=\sigma_{A} \exp -\frac{E_{A}}{k_{B} T}
$$

where $k_{\mathrm{B}}$ is Boltzmann's constant, $\sigma_{\mathrm{A}}$ is another prefactor, and $T_{\mathrm{A}}$ is a characteristic temperature for the thermally activated process with an activation energy of $\mathrm{E}_{\mathrm{A}}$.

It follows that one process alone cannot fit the whole $T$ range, as previously assumed [32,37]. At low $T$, VRH prevails,

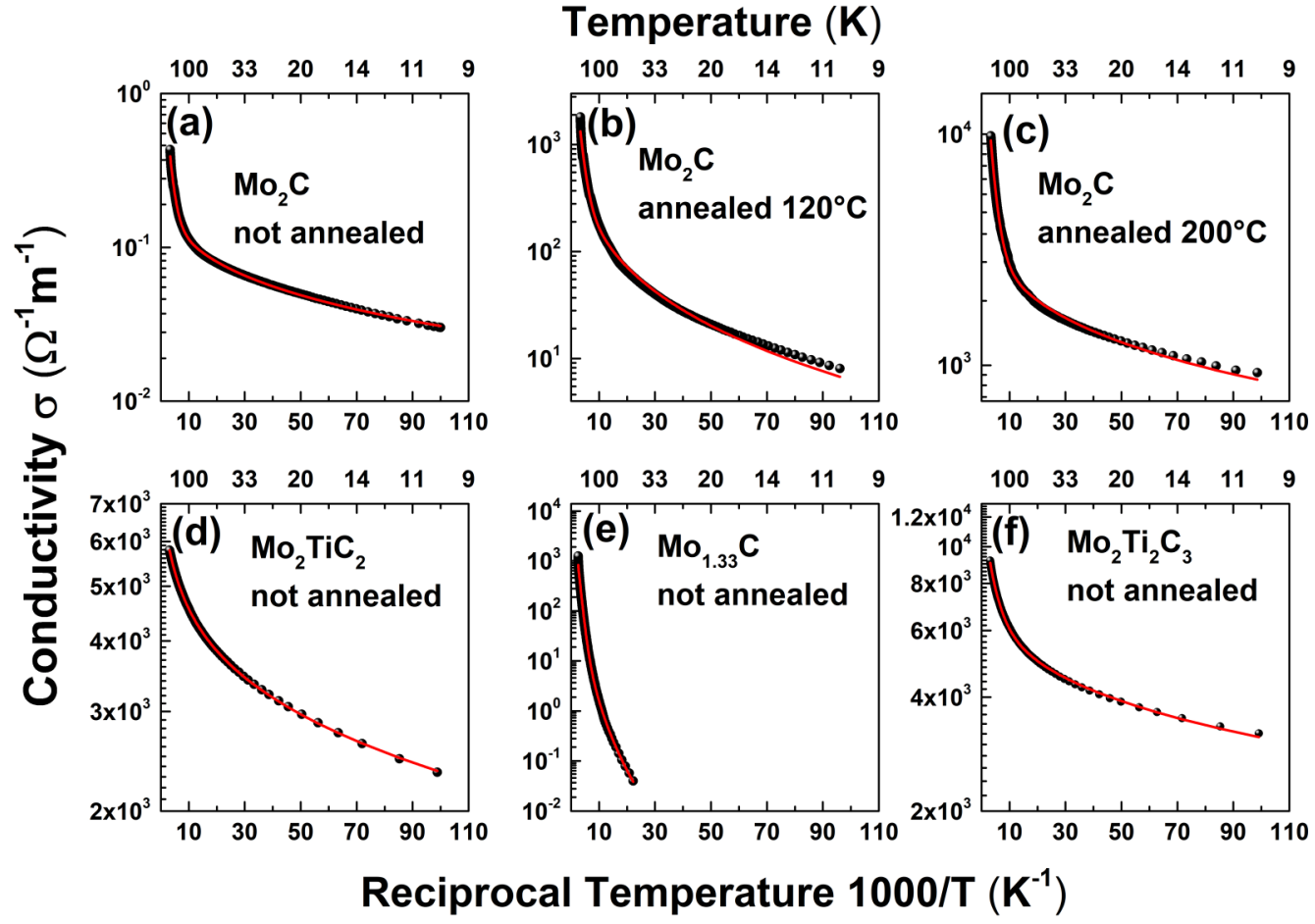

FIG. 6. Conductivity (log scale) vs $1000 / \mathrm{T}$ of delaminated, filtered, freestanding thin films of (a) $\mathrm{Mo}_{2} \mathrm{CT}_{z} \mathrm{unannealed}$ (b) $\mathrm{Mo}_{2} \mathrm{CT}_{z}$ annealed at $120{ }^{\circ} \mathrm{C}$, (c) $\mathrm{Mo}_{2} \mathrm{CT}_{z}$ annealed at $200{ }^{\circ} \mathrm{C}$, (d) $\mathrm{Mo}_{2} \mathrm{TiC}_{2} \mathrm{~T}_{z}$ unannealed, (e) $\mathrm{Mo}_{1.33} \mathrm{CT}_{z}$ unannealed, and (f) $\mathrm{Mo}_{2} \mathrm{Ti}_{2} \mathrm{C}_{3} \mathrm{~T}_{z}$ unannealed. Data are shown as solid circles. Parameters for the fits, given by solid red lines, are listed in Table II assuming a thermally activated model and a VRH component with $x=1 / 4$ (3D VRH). Parameters of fits for $x=1 / 2$ are also listed in Table II and were equally good. Those for $x=1 / 3$ can be found in Table S1 in Supplemental Material. 
TABLE II. Parameters obtained by fitting experimental results—-shown as solid circles in Fig. 6—assuming Eqs. (1)-(4) are valid. All conductivity values are in $\mathrm{S} / \mathrm{m}$.

\begin{tabular}{|c|c|c|c|c|c|c|c|c|c|c|}
\hline Sample & $\sigma_{0}(\mathrm{~S} / \mathrm{m})$ & $\mathrm{T}_{0}(\mathrm{~K})$ & $\sigma_{\mathrm{A}}(\mathrm{S} / \mathrm{m})$ & $\mathrm{E}_{\mathrm{A}}(\mathrm{eV})\left(\mathrm{T}_{\mathrm{a}}\right)$ & $\sigma_{0}(\mathrm{~S} / \mathrm{m})$ & $\mathrm{T}_{0}(\mathrm{~K})$ & $\sigma_{\mathrm{A}}(\mathrm{S} / \mathrm{m})$ & $\mathrm{E}_{\mathrm{A}}(\mathrm{eV})\left(\mathrm{T}_{\mathrm{a}}\right)$ & Comments & Ref. \\
\hline $\begin{array}{l}\text { Annealed at } \\
393 \mathrm{~K} 18 \mathrm{~h}\end{array}$ & 9835 & $2.93 \times 10^{4}$ & 8500 & $0.58(673.3 \mathrm{~K})$ & 591.4 & 221.3 & 6191 & $0.047(543.2 \mathrm{~K})$ & & {$[32]$} \\
\hline \multicolumn{11}{|c|}{$\mathrm{Mo}_{1.33} \mathrm{CT}_{z}$} \\
\hline Unannealed & $2.84 \times 10^{7}$ & $7.8 \times 10^{6}$ & $1.74 \times 10^{4}$ & $0.11(1310 \mathrm{~K})$ & 2443 & 5547 & $1.37 \times 10^{4}$ & $0.10(1182 \mathrm{~K})$ & & This paper \\
\hline Unannealed & $3.9 \times 10^{9}$ & $4.5 \times 10^{7}$ & 3411 & $0.14(1576.8 \mathrm{~K})$ & 9104 & 15301 & 4485 & $0.13(1507 \mathrm{~K})$ & & [28] \\
\hline \multicolumn{11}{|c|}{$\mathrm{Mo}_{2} \mathrm{TiC}_{2} \mathrm{~T}_{z}$} \\
\hline Unannealed & $1.03 \times 10^{4}$ & 48 & 443.6 & $0.015(172.1 \mathrm{~K})$ & 5610 & 7.9 & 1605 & $0.014(161 \mathrm{k})$ & & This paper \\
\hline \multicolumn{11}{|c|}{$\mathrm{Mo}_{2} \mathrm{Ti}_{2} \mathrm{C}_{3} \mathrm{~T}_{z}$} \\
\hline Unannealed & $1.27 \times 10^{4}$ & 38.2 & 4847 & $0.0224(279 \mathrm{k})$ & 7200 & 7.3 & 5929 & $0.02(238 \mathrm{~K})$ & & This paper \\
\hline Unannealed & 4320 & 206.2 & 980 & $0.03(332 \mathrm{~K})$ & 1922 & 19.52 & 1246 & $0.021(248 \mathrm{~K})$ & $\begin{array}{c}\text { Multilayered } \\
\text { cold pressed } \\
\text { disks }\end{array}$ & [37] \\
\hline
\end{tabular}

whereas at higher $T$ transport is thermally activated. That said it is important to note that in many cases, even at RT, the majority of the current is carried by VRH carriers.

The rationale for assuming VRH and thermal activation is that both relationships are characteristic of transport in disordered conductive media [41-50]. In such systems, $\mathrm{E}_{\mathrm{F}}$ is positioned in a band of localized states, separated from delocalized ones, that appear at higher energies, by a threshold referred to as the mobility edge, $\mathrm{E}_{\mathrm{C}}$ [53]. Around $\mathrm{E}_{\mathrm{F}}$, transport is by phonon-assisted hopping, and the hopping rate is determined by a balance between tunneling probability, the activated term (including the phonon energy gain), and the DOS available for hopping [53]. This balance determines a T-dependent preferential hopping distance, $\lambda$, responsible, in turn, for the characteristic form of Eq. (1) [53]. At higher temperatures, transport is by delocalized electrons thermally excited above $E_{C}$ [53]. Since these two processes act in parallel their contributions to $\sigma$ can be summed up as in Eq. (3).

The phenomenon - in which $\sigma$ undergoes a discontinuous Mott transition, when crossing $\mathrm{E}_{\mathrm{C}}$ - has been the subject of controversy to this day [56]. According to the Ioffe-Regel principle, $\sigma$ at the threshold equals a minimum conductivity value, $\sigma_{\min }$, proportional to the intersite distance, $\delta$, in three-dimensional (3D) systems [53]. However, at lower T, quantum effects start to prevail and may give rise to additional interference regimes, that in turn invalidate the concept of $\sigma_{\text {min }}$ [57]. However, in the relatively high-temperature thermally activated regime, quantum interference effects can be safely excluded and the concept of minimum conductivity applies. Said otherwise, the temperature range of our data clearly excludes a quantum effects regime, which indicates that VRH is prevalent at low T. However, as noted, above a certain T, the number of thermally excited carriers, above $\mathrm{E}_{\mathrm{C}}$, is large enough for $\sigma$ to fall in the thermally activated regime [53].

Figures 6(a)-6(f) plot $\log \sigma_{\text {tot }}$ versus $1 / \mathrm{T}$ for, respectively, unannealed $\mathrm{Mo}_{2} \mathrm{CT}_{z}, \mathrm{Mo}_{2} \mathrm{CT}_{z}$ annealed at $393 \mathrm{~K}$ and $473 \mathrm{~K}$, and unannealed $\mathrm{Mo}_{2} \mathrm{TiC}_{2} \mathrm{~T}_{z}, \mathrm{Mo}_{1.33} \mathrm{CT}_{z}$, and $\mathrm{Mo}_{2} \mathrm{Ti}_{2} \mathrm{C}_{3} \mathrm{~T}_{z}$. In all cases, the data points were fit using a Levenberg-Marquardt algorithm—shown by solid red lines—assuming Eqs. (1)-(4) are valid. In Fig. 5(a), $x$ is assumed to be $1 / 4$ (i.e., a 3D VRH is assumed). The fitting parameters for $x=1 / 4$ and $1 / 2$ are listed in Table II. The fitting parameters for $x=1 / 3$ are listed in Table S1 in the Supplemental Material [35]. However, since, as argued below, the behavior of our films is reminiscent of granular metals, the exponent $1 / 2$ should not be dismissed, because charge effects in metallic flakes can be important $[41,50]$. Note that for a given composition the choice of $\mathrm{x}$ does not greatly affect $\mathrm{E}_{\mathrm{a}}$. Equally important, it is difficult to differentiate between the various $\mathrm{x}$ values, which renders it difficult to unambiguously determine the value of the exponent $x$. Herein, we narrow down the possible mechanisms to three: 2D, 3D VRH, and the Efros-Shklovskii model.

In Mott's model, $\mathrm{T}_{0}$ is related to $\mathrm{D}\left(\mathrm{E}_{\mathrm{F}}\right)$ by a relation of the form [53]

$$
T_{0}=\frac{a}{k_{B} D\left(E_{F}\right) \gamma^{-3}}
$$

where $1 / \gamma$ is the decay length or reciprocal tunneling exponent of the localized states. $\mathrm{D}\left(\mathrm{E}_{\mathrm{F}}\right) \gamma^{-3}$ is called the localization parameter; $a$ is a constant initially estimated by Mott to be 16, which later, more accurate, numerical calculations showed to be 64 [58]. Equation (5) applies to a system where $\mathrm{D}\left(\mathrm{E}_{\mathrm{F}}\right)$ does not vary much in the vicinity of $\mathrm{E}_{\mathrm{F}}$, at least over a range $\approx \mathrm{k}_{\mathrm{B}} \mathrm{T}$. In the case of an exponential DOS $a$ was 

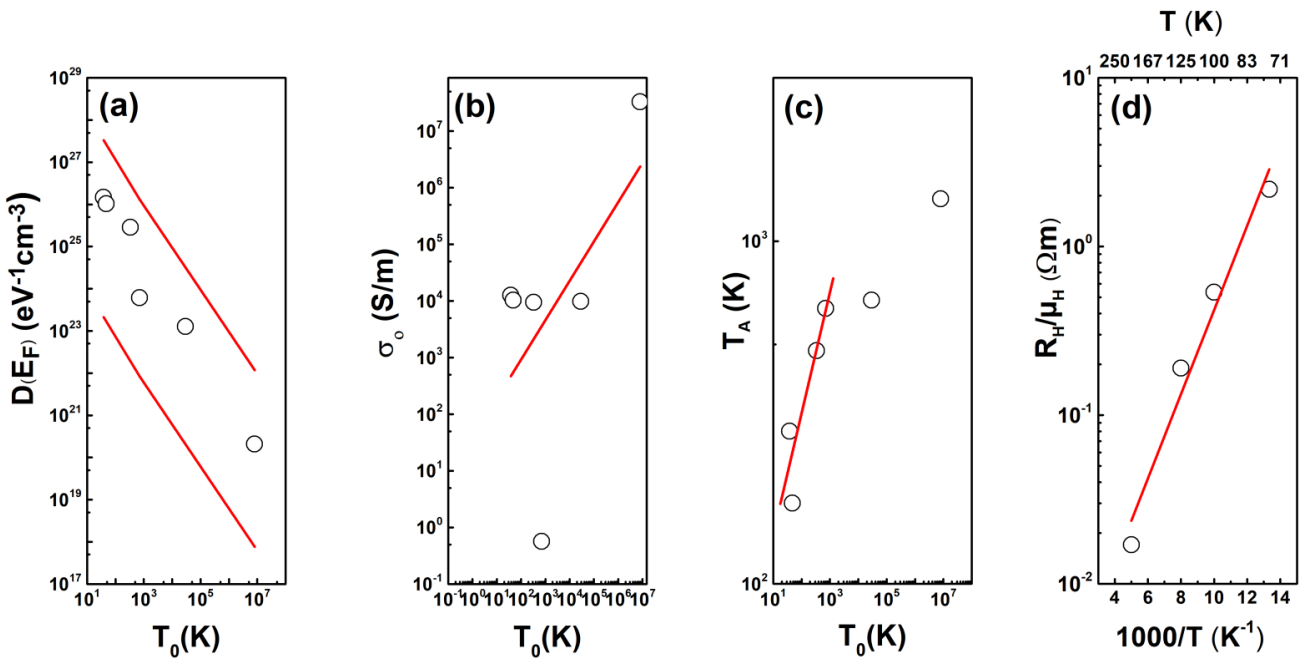

FIG. 7. (a) Apparent density of states at $\mathrm{E}_{\mathrm{F}}$, extracted from Eq. (5), vs $\mathrm{T}_{0}$. The solid lines represent the expected values assuming either $1 / \gamma=0.2$ or $5 \mathrm{~nm}$, and $\mathrm{a}=310$. (b) Variation of $\sigma_{0}$ with $\mathrm{T}_{0}$; the solid line is a linear fit of the data points. (c) Variation of $\mathrm{T}_{\mathrm{A}}$ with $\mathrm{T}_{0}$; the solid line is a guide for the eye, illustrating that a correlation exists between $\mathrm{T}_{\mathrm{A}}$ and $\mathrm{T}_{0}$ when $\mathrm{T}_{0}$ lies in the range characteristic of the granular metal hopping transport. (d) Variation of $\mathrm{R}_{\mathrm{H}} / \mu \mathrm{H}$ vs reciprocal temperature in the case of $\mathrm{Mo}_{1.33} \mathrm{CT}_{z}$.

found to exceed 300 [58]. In the case of "homogeneous" disordered systems such as amorphous semiconductors (a-Si, a-Ge, etc.), carbonized polymers, noncrystalline chalcogenide compounds, etc., $\mathrm{D}\left(\mathrm{E}_{\mathrm{F}}\right)$ is of order $10^{18}-10^{19} \mathrm{eV}^{-1} \mathrm{~cm}^{-3}$ [see Ref. [58] and references therein, and Refs. [59-61]]. When these $\mathrm{D}\left(\mathrm{E}_{\mathrm{F}}\right)$ values are combined with typical, but physically sound, $\gamma$ values of the order of $0.3-10 \mathrm{~nm}$ [41], the estimated $\mathrm{T}_{0}$ values are well above $10^{5}$ and can rise to values as high as $10^{12} \mathrm{~K}$ [58]. For example, intraflake VRH has been observed in $2 \mathrm{D} \mathrm{MoS}_{2}$, in which case $\mathrm{T}_{0}$ was found to exceed $10^{4} \mathrm{~K}$ [60] and was attributed to an impurity band conduction induced by sulphur vacancies, forming a 2D equivalent of a 3D amorphous semiconductor. From the results listed in Table II, it is clear that only the $\mathrm{T}_{0}$ values for the $\mathrm{Mo}_{1.33} \mathrm{C}$ films are in the $10^{7}-\mathrm{K}$ regime. This composition is unique in that every third atomic site is vacant [28]. In other words, this composition is highly defective and based on its $\mathrm{T}_{0}$ value it is reasonable to assume that in this composition VRH is occurring within each flake.

We now return to the other compositions. As seen from Table II, with the notable exceptions of $\mathrm{Mo}_{1.33} \mathrm{CT}_{z}-$ discussed separately below-all other $\mathrm{T}_{0}$ values lie in the $10-10^{4}-\mathrm{K}$ range. The latter values are thus incompatible with a system where $\mathrm{D}\left(\mathrm{E}_{\mathrm{F}}\right)$ is "low." These low $\mathrm{T}_{0}$ values are in much better agreement with those found in granular metals and cermets. In such "inhomogeneous" materials, the $\mathrm{T}_{0}$ 's usually range from 1 to $10^{4} \mathrm{~K}$ [41-47], and the hopping is controlled by tunneling across thin insulating layers separating the highly metallic islands/grains [42], or the thin amorphous insulating films in the case of cermets [41,49]. Since in our materials it is reasonable to assume that the interflake space is disordered and DFT calculations confirm a large $\mathrm{D}\left(\mathrm{E}_{\mathrm{F}}\right)$ [40], we follow the lead of such models.

Figure 7(a) shows a plot of the variation of $\mathrm{D}\left(\mathrm{E}_{\mathrm{F}}\right)$ expected from the extracted $\mathrm{T}_{0}$ values, assuming Eq. (5) is valid, $\mathrm{a}=$ 310 , and $\gamma$ is between 0.2 and $5 \mathrm{~nm}$ [58]. Note that the range chosen for $\gamma$ covers the experimentally measured $\mathrm{d}_{\mathrm{c} / 2}$ values (Table I). With the exception of $\mathrm{Mo}_{1.33} \mathrm{CT}_{z}$, all other $\mathrm{D}\left(\mathrm{E}_{\mathrm{F}}\right)$ values calculated are $>10^{23} \mathrm{eV}^{-1} \mathrm{~cm}^{-3}$. These values are incompatible with values expected for localized impurity or defect bands inside band gaps of amorphous semiconductors. Although it should be noted that the extracted values should certainly not be seen as a reliable estimate of a flake's DOS, they are connected to it, so that it may be interesting to sort the various compounds in decreasing order of this estimation: $\mathrm{Mo}_{2} \mathrm{Ti}_{2} \mathrm{C}_{3} \mathrm{~T}_{z}, \mathrm{Mo}_{2} \mathrm{TiC}_{2} \mathrm{~T}_{z}, \mathrm{Mo}_{2} \mathrm{CT}_{z}$, and $\mathrm{Mo}_{1.33} \mathrm{CT}_{z}$.

A second quantity of interest is the conductivity prefactor, $\sigma_{0}$. Whereas variations in $\mathrm{T}_{0}$ can be ascribed to variations in $\gamma$, the correlation between $\sigma_{0}$ and $\mathrm{T}_{0}$ depends in a complex way on the DOS profile: a constant DOS should lead to a slow decrease of $\sigma_{0}$ with $\mathrm{T}_{0}$, whereas an exponential tail of localized states leads to an exponential increase of $\sigma_{0}$ with $\mathrm{T}_{0}$ [58]. Figure 7(b) is a plot of $\sigma_{0}$ versus $\mathrm{T}_{0}$ which does not show a clear correlation between $\sigma_{0}$ and $\mathrm{T}_{0}$. This may be due to the fact that is some samples the percolation effects affect the prefactor without affecting the characteristic temperature $\mathrm{T}_{0}$, as explained later in the discussion about the conductivity prefactor.

Thirdly, a correlation between the $T_{0}$ and $T_{A}$ values is evidenced in Fig. 7(c). Such a correlation has been observed in cermets [49].

The $\mathrm{E}_{\mathrm{A}}$ values reported here (Table II) can be grouped into three groups.

(i) $\mathrm{Mo}_{2} \mathrm{Ti}_{2} \mathrm{C}_{3} \mathrm{~T}_{z}$ and $\mathrm{Mo}_{2} \mathrm{Ti}_{2} \mathrm{CT}_{z}$, exhibit the lowest values, of order $20 \mathrm{meV}$ (Table II).

(ii) $\mathrm{Mo}_{2} \mathrm{CT}_{z}$ exhibits values of order $40 \mathrm{meV}$, after annealing, while the preannealing value was several $\mathrm{meV}$ higher. Here, $E_{\mathrm{A}}$ depends slightly on the value of $\mathrm{x}$ chosen to fit the data.

(iii) The highest $\mathrm{E}_{\mathrm{A}}$ values, exceeding $0.1 \mathrm{eV}$, were observed for $\mathrm{Mo}_{1.33} \mathrm{CT}_{z}$, irrespective of the $\mathrm{x}$ value chosen for fitting.

Although such values may be seen to be quite low if the energy barrier determining the mobility edge corresponds to an interflake potential, it is worth noting that highly metallic flakes could lead to appreciable image forces, thereby 
TABLE III. Hall effect, charge-carrier concentration, and mobility of Mo-based delaminated freestanding thin films at various temperatures.

\begin{tabular}{|c|c|c|c|c|c|}
\hline Sample & Temp. (K) & $\mathrm{R}_{\mathrm{H}}\left(10^{-15} \mathrm{~cm}^{3} / \mathrm{C}\right)$ & Carrier concentration $\left(10^{20} \mathrm{~cm}^{-3}\right)$ & Mobility $\left(\mathrm{cm}^{2} / \mathrm{V} \mathrm{s}\right)$ & Ref. \\
\hline \multirow[t]{2}{*}{$\mathrm{Mo}_{2} \mathrm{CT}_{z}$ annealed $200^{\circ} \mathrm{C} 18 \mathrm{~h}$} & 10 & $-400 \pm 60$ & $0.16 \pm 0.02(\mathrm{n})$ & $3.7 \pm 0.5$ & This paper \\
\hline & 150 & $-8 \pm 6$ & $7.9 \pm 0.1(\mathrm{n})$ & $0.3 \pm 0.2$ & This paper \\
\hline \multirow[t]{3}{*}{$\mathrm{Mo}_{1.33} \mathrm{CT}_{z}$ unannealed } & 75 & $-96,000 \pm 1,000$ & $0.00065 \pm 0.0005(\mathrm{n})$ & $0.44 \pm 0.05$ & This paper \\
\hline & 100 & $-44,443 \pm 110$ & $0.0014 \pm 0.0001(\mathrm{n})$ & $0.83 \pm 0.02$ & This paper \\
\hline & 125 & $-13,318 \pm 300$ & $0.0047 \pm 0.0001(\mathrm{n})$ & $0.70 \pm 0.01$ & This paper \\
\hline \multirow[t]{2}{*}{$\mathrm{Mo}_{2} \mathrm{Ti}_{2} \mathrm{C}_{3} \mathrm{~T}_{z}$ unannealed } & 40 & $6.6 \pm 0.1$ & $9.49 \pm 0.02(\mathrm{p})$ & $0.31 \pm 0.01$ & This paper \\
\hline & 200 & $3.76 \pm 0.07$ & $16.63 \pm 0.03(\mathrm{p})$ & $0.29 \pm 0.01$ & This paper \\
\hline $\mathrm{Mo}_{2} \mathrm{Ti}_{2} \mathrm{C}_{3} \mathrm{~T}_{z}$ unannealed & 300 & & $2.01(\mathrm{n})$ & 0.45 & [24] \\
\hline $\mathrm{Mo}_{2} \mathrm{Ti}_{2} \mathrm{C}_{3} \mathrm{~T}_{z}$ Ann. at $800 \mathrm{~K}$ in $\mathrm{Ar} / \mathrm{H}_{2}$ & 300 & & $18.3(\mathrm{n})$ & 2.05 & [24] \\
\hline \multirow[t]{2}{*}{$\mathrm{Mo}_{2} \mathrm{TiC}_{2} \mathrm{~T}_{z}$ unannealed } & 40 & $-2.84 \pm 0.01$ & $22.0 \pm 0.1(\mathrm{n})$ & $0.1 \pm 0.01$ & This paper \\
\hline & 200 & $-3.21 \pm 0.03$ & $19.4 \pm 0.2(\mathrm{n})$ & $0.17 \pm 0.01$ & This paper \\
\hline
\end{tabular}

reducing the effective potential value, as already hypothesized in the case of granular metals [41]. In the case of unannealed samples, the additional presence of cations between the layers is expected to reduce the interflake potential if the carriers are electrons.

The Ioffe-Regel principle stipulates that in highly disordered systems the mean free path cannot be smaller than the interatomic distance $\delta$, so that $\lambda_{\min } \sim \delta$ or, alternatively, this minimum value is fixed by the condition $\mathbf{k}_{\mathrm{F}} \lambda_{\min } \sim 1$, where $\mathbf{k}_{\mathrm{F}}$ the Fermi wave vector [53]. This minimum conductivity, $\sigma_{\min }$, is given by $0.026 \mathrm{e}^{2} / \hbar \delta$ in a $3 \mathrm{D}$ system with a coordination number of 6 . A spectacular manifestation of this principle is the saturation of the resistivity of some conventional metals observed just before melting [62]. In highly disordered systems, and again assuming $\delta$ in the 0.3 - to $5-\mathrm{nm}$ range, $\sigma_{\min }$ values are in the range of 1000 to $30000 \mathrm{~S} / \mathrm{m}$ [53]. Comparing this range to values calculated herein (e.g., column 4 in Table II) shows that, with one exception, the $\sigma_{\mathrm{A}}$ values fall in the above-calculated range. Departures - clearly not observed here-may be due to the observation of the Meyer-Neldel rule, consisting in an exponential dependence of $\sigma_{\mathrm{A}}$ on $\mathrm{E}_{\mathrm{A}}$, most often attributed to a low and rapidly varying DOS around $\mathrm{E}_{\mathrm{F}}$ [63], also observed with VRH [58,59].

In the case of the unannealed $\mathrm{Mo}_{2} \mathrm{CT}_{z}$ sample, $\sigma_{\mathrm{A}}$ falls below any reasonable limit in the frame of a $\sigma_{\min }$ concept. At room temperature, both the VRH mechanism and activated transport substantially contribute to the overall conductivity. The fact that $\sigma$ of this material decreases exponentially with increasing $d_{c / 2}$ [Fig. 4(a)] is a strong indication that here again tunneling between the layers is the operative mechanism for the VRH component. Note that in this system the volume fraction and size distribution of the flakes can be assumed to be more or less the same, the only variable being $d_{c / 2}$. Due to the voids between flakes (Fig. 1), the sample cannot be considered as homogeneous at the microscopic level but is probably subject to percolation $[48,64,65]$. Since here the MXene volume is clearly not equal to the total volume (see Fig. 1), it is reasonable to assume that porosity could substantially affect the conductivity. Percolation is compatible with a conductivity prefactor that is $\ll \sigma_{\min }$, while maintaining an activation energy $\mathrm{E}_{\mathrm{A}}$ close to, but higher than, the annealed $\mathrm{Mo}_{2} \mathrm{C}$ samples (see Table I), since the current in the percolation paths still has to be controlled by the same kind of activated process. All the aforementioned considerations are justified on the basis of numerical calculations in a system somewhat similar to the one explored here, but for the fact that flakes are replaced by nanowires [65]. In Ref. [65], it is also shown that in the case of complex percolation paths one can still consider both VRH and activated regimes as acting in parallel.

We now turn our attention to the Hall effect results, shown in Table III in which a single band model is assumed and $\sigma$ is deconvoluted into a carrier density, $\mathrm{n}$, and mobility, $\mu$. These values are simply listed for the sake of completeness since as discussed below, except in the thermally activated regime, they have to be considered, at the very best, "effective" or curve fitting values, with little physical basis.

In the VRH regime, Hall data cannot be used to estimate electron concentrations [53] for the simple reason that in most disordered systems it is unlikely that a simple relation connecting $\mathrm{D}\left(\mathrm{E}_{\mathrm{F}}\right)$ to the total carrier concentration in a band exists. The latter is a prerequisite for relating transport properties to electron concentrations. In some systems, the Hall effect is not even measurable [53], but many hopping processes are assumed to favor the observation of a thermally activated Hall mobility $[53,66]$. In our case, the situation is complicated by the fact that even perfectly metallic MAX phases and MXenes most often combine electron and hole pockets at the Fermi level, associated with complex Fermi-surface shapes [67].

In the thermally activated regime, however, the situation is slightly different in that if one assumes a relation such as $R_{\mathrm{H}} / \mu_{\mathrm{H}}=\sigma_{\min }{ }^{-1} \exp \left(\mathrm{E}_{\mathrm{C}}-\mathrm{E}_{\mathrm{F}}\right) / \mathrm{kT}=\sigma_{\min }{ }^{-1} \exp -\mathrm{E}_{\mathrm{A}} / \mathrm{kT}$ then a plot of $R_{\mathrm{H}} / \mu_{\mathrm{H}}$ versus $1 / \mathrm{T}$ should yield a straight line with a slope of $\mathrm{E}_{\mathrm{A}}$. Making use of the $\mathrm{Mo}_{1.33} \mathrm{C}$ results at the higher temperatures listed in Table III, from the slope of an Arrhenius plot [Fig. 7(d)] an $\mathrm{E}_{\mathrm{A}}$ of $0.05 \mathrm{eV}$ is obtained, which is slightly lower than the $\mathrm{E}_{\mathrm{A}}$ values $(\approx 0.1 \mathrm{eV})$ determined in Table II. This is one more argument in favor of a thermally activated regime in the highest $\mathrm{T}$ range.

Interpreting MR results in disordered systems is still an elusive domain, where it is rare to find quantitative and 

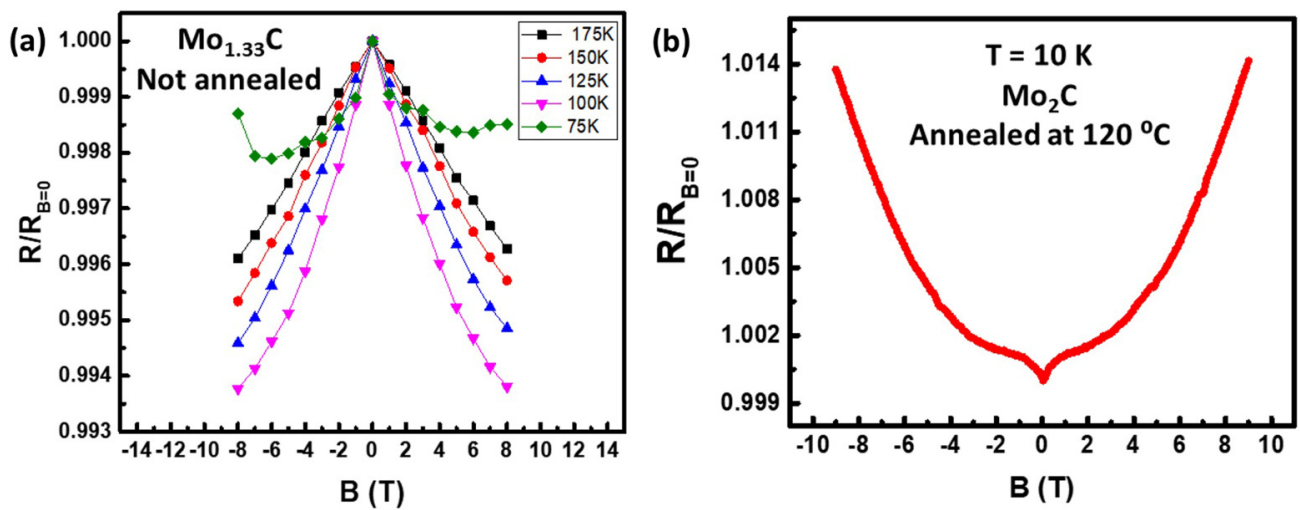

FIG. 8. Magnetoresistance (MR) data at various temperatures for (a) $\mathrm{Mo}_{1.33} \mathrm{CT}_{z}$ not annealed and (b) $\mathrm{Mo}_{2} \mathrm{CT}_{z}$ annealed at $120{ }^{\circ} \mathrm{C}$ delaminated freestanding thin films.

satisfying comparisons between experiment and theory except in the case of weak localization (WL) [68]. MR in hopping systems can be positive if magnetic-field-induced shrinking of the localized waves prevails [53], negative, and sometimes predicted to be linear [69-72]. Some argue that WL should not be observed or be much reduced in such systems (see, e.g., Ref. [61]). However, the MRs we observed are, in all cases, quite small, so that we cannot exclude the fact that what we actually observe are intraflake WL phenomena. Depending on sample and temperature, we actually observed both positive and negative MR (Fig. 8). However, our data do not allow us to identify any of the possible underlying mechanisms with certainty.

We have no good explanation for the result of the $\mathrm{Mo}_{2} \mathrm{CT}_{z}$ sample annealed at $200^{\circ} \mathrm{C}$, where both $R_{\mathrm{H}}$ and $V_{\mathrm{H}}$ vary substantially with $\mathrm{T}$ between 10 and $150 \mathrm{~K}$. In contrast to the other samples, this may be due to the fact that the Hall data at $10 \mathrm{~K}$ reflect an increasing influence of the VRH regime. We stress that in all cases unfortunately neither "real" carrier densities nor "real" carrier mobilities can be reliably extracted from the MR data, and most of all it is virtually impossible to extrapolate to the carrier densities of perfect flakes. However, effective Hall carrier densities and Hall mobilities can only be used for the sake of quantitative comparisons between different samples.

One of the important conclusions of our paper is that the nature of the terminations on conductivity is secondary when compared with interlayer spacing. This is best seen in Fig. 4 where we plot conductivity versus interlayer spacing [Fig. 4(a)] and annealing temperature [Fig. 4(b)]. In general, it is nontrivial to change the nature of the terminations by heating even in ultrahigh vacuum. For example, heating $\mathrm{Ti}_{3} \mathrm{C}_{2}$ MXene up to $550^{\circ} \mathrm{C}(823 \mathrm{~K})$ in high vacuum did not visibly alter their x-ray photoelectron spectroscopy (XPS) spectra [73]. Based on this information, it is reasonable to assume that the surface terminations of $\mathrm{Mo}_{2} \mathrm{CT}_{z}$ did not vary much when the material was annealed at $473 \mathrm{~K}$. And yet the conductivity was enhanced by more than five orders of magnitude [Fig. 4(a)], and the change was correlated to changes in interlayer spacing. It is for these reasons that we believe the exact nature of the terminations to be secondary to interlayer spacing. At some level, this is not too surprising since for the electrons to hop from flake to flake they would have to go through the terminations as well as whatever is in between the layers.

Equally important, Kim et al. [24] showed that after annealing their $\mathrm{Mo}_{2} \mathrm{CT}_{z}, \mathrm{Mo}_{2} \mathrm{TiC}_{2} \mathrm{~T}_{z}$, and $\mathrm{Mo}_{2} \mathrm{Ti}_{2} \mathrm{C}_{3} \mathrm{~T}_{z}$ samples in $\mathrm{Ar} / \mathrm{H}_{2}$ gas at $800 \mathrm{~K}$ the nature of the conductivity in the former two became metalliclike. This observation is totally consistent with our original conjecture that the flakes themselves remain metallic in nature and that it is only what is between the layers that determines the nature of the conductivity, viz., VRH or metallic. This is an important conclusion that cannot be easily dismissed.

Examining the interlayer distances after annealing at $800 \mathrm{~K}$ we find that in the case of $\mathrm{Mo}_{2} \mathrm{CT}_{z}$, at $0.79 \mathrm{~nm}, \Delta \mathrm{d}_{002}-$ defined as the actual interlayer distance minus the value it would have been in the absence of any interlayer molecules such as water [74] - is almost nil [24]. In other words, the interlayer distance after annealing is almost identical to the one expected for $\mathrm{Mo}_{2} \mathrm{CO}_{2}$, with no molecules in between the layers as predicted by DFT [75]. It is thus tempting to ascribe the change in mechanism-from VRH to metallic-to the lack of interlayer molecules. That conclusion, however, is belied by the fact that the same change in mechanism is also seen in the $\mathrm{Mo}_{2} \mathrm{TiC}_{2} \mathrm{~T}_{z}$ films. In that case, at $0.295 \mathrm{~nm}$, $\Delta \mathrm{d}_{002}$ is consistent with the presence of at least one water layer and/or TBAOH molecules in the interlayer space. This comment notwithstanding these results show that, at least for $\mathrm{Mo}_{2} \mathrm{CT}_{z}$ and $\mathrm{Mo}_{2} \mathrm{TiC}_{2} \mathrm{~T}_{z}$, the presence of terminations and molecules between the layers is not an impediment to obtain metal-like conductivity.

Interestingly, the conductivity of the $\mathrm{Mo}_{2} \mathrm{Ti}_{2} \mathrm{C}_{3} \mathrm{~T}_{z}$ sample did not become metallic after the $800-\mathrm{K}$ annealing, but instead became much less sensitive to temperature [24]. Note that at $\approx 0.2 \mathrm{~nm} \Delta \mathrm{d}_{002}$ is lower than for the $\mathrm{Mo}_{2} \mathrm{TiC}_{2} \mathrm{~T}_{z}$ films. The reason for this state of affairs is unclear at this time, but one possibility is that this composition was not sufficiently annealed and that had it been annealed for long enough it too would also have become a metal-like conductor. More work, some of which is underway, is needed to understand the important transition from VRH to metalliclike conductivity. This question is not only important from a fundamental point of view, but also of great practical import since, in many applications, it is important to maximize the conductivity. 
Lastly, we consider the role of surface terminations. In general, at least theoretically, many papers exist that claim that the $\mathrm{D}\left(\mathrm{E}_{\mathrm{F}}\right)$ can be varied by changing the nature of the terminations. As determined by XPS, the chemistry of unannealed $\mathrm{Mo}_{2} \mathrm{CT}_{z}$ is $\mathrm{Mo}_{2} \mathrm{C}_{0.9} \mathrm{O}_{1.0}(\mathrm{OH})_{1.2} \mathrm{~F}_{0.1} 0.4 \mathrm{H}_{2} \mathrm{O}_{\text {ads }}$ [32]. The chemistry of $\mathrm{Mo}_{1.33} \mathrm{CT}_{z}$ on the other hand is $\mathrm{Mo}_{1.2} \mathrm{CO}_{0.7}(\mathrm{OH})_{0.5} \mathrm{~F}_{1.1} 0.4 \mathrm{H}_{2} \mathrm{O}_{\text {ads }}$ [40]. It follows that the latter has significantly more $\mathrm{F}$ terminations than the former. In all cases, however, DFT calculations have shown that, at least for these two compounds, $\mathrm{D}\left(\mathrm{E}_{\mathrm{F}}\right)$ is substantial for termination chemistries that are close to those determined by XPS [40]. The fact that after annealing at $550^{\circ} \mathrm{C}$ under vacuum results in some cases in metalliclike conductivity is fully consistent with our conjecture that the conductivity is controlled by the interlayer distance.

This does not, however, imply that there are no other factors. The overall situation is not that simple since the sensitivity of the resistivity to interlayer spacing depends on MXene chemistry. For example, consider $\mathrm{Ti}_{3} \mathrm{C}_{2} \mathrm{~T}_{z}$ and $\mathrm{Mo}_{2} \mathrm{CT}_{z}$; in the former, increasing $\Delta \mathrm{d}_{002}$ from $\approx 0.46$ to $\approx 2.8$ $\mathrm{nm}$ only increases the resistivity from $\approx 0.3$ to $4.5 \Omega \mathrm{cm}$ [76]. In the latter, increasing $\Delta \mathrm{d}_{002}$ from $\approx 0.46$ to $1.1 \mathrm{~nm}$ increases the resistivity by over three orders of magnitude [Fig. 4(a)]. Equally important, the conductivity of $\mathrm{Ti}_{3} \mathrm{C}_{2} \mathrm{~T}_{z}$ is mostly metallic in nature. Another complication to the idea that interlayer spacing solely determines electronic transport is the fact that, in general, the higher the value of $n$ in $\mathrm{M}_{n+1} \mathrm{C}_{n} \mathrm{~T}_{z}$, the more conductive the films for the same $\Delta \mathrm{d}_{002}$ and the less sensitive the conductivity is to $\Delta \mathrm{d}_{002}$ [Fig. $4(\mathrm{a})$ ]. In short, more detailed work is needed, and indicated, to better understand the intricacies of electronic transport in MXenes.

\section{CONCLUSIONS}

All Mo-based MXenes tested herein obey transport laws specific to disordered systems. Two transport regimes acting in parallel manifest themselves; at low temperatures, VRH is applicable; at higher temperatures, the transport is partially thermally activated. A quantitative analysis of the two regimes shows that - with the exception of the highly defective $\mathrm{Mo}_{1.33} \mathrm{CT}_{z}$ composition-our data compare well with the literature of hopping transport in granular metals and cermets, as opposed to "homogeneous" amorphous semiconductors. This conclusion clearly favors electrical transport limited by interflake transfer at the microscopic level and is consistent with the fact that $\sigma$ is a strong function of $\mathrm{d}_{\mathrm{c} / 2}$ [Fig. 4(a)].

\section{ACKNOWLEDGMENTS}

We acknowledge Dr. B. Anasori at Drexel University for providing the $\mathrm{Mo}_{2} \mathrm{Ti}_{2} \mathrm{C}_{3}$ and $\mathrm{Mo}_{2} \mathrm{TiC}_{2}$ samples. We also acknowledge both $\mathrm{V}$. Natu and $\mathrm{Q}$. Tao for providing $\mathrm{Mo}_{2} \mathrm{Ga}_{2} \mathrm{C}$ and $\left(\mathrm{Mo}_{2 / 3} \mathrm{Sc}_{1 / 3}\right)_{2} \mathrm{AlC}$ powders, respectively. S. Kota is acknowledged for his help and efforts synthesizing $\mathrm{Mo}_{1.33} \mathrm{C}$ and $\mathrm{Mo}_{2} \mathrm{C}$ freestanding films. M.W.B. acknowledges the support of the Chair of Excellence from UGA Nanosciences Foundation, Grenoble, France. T.O. was supported by the Flag-ERA JTC 2-17 project entitled "MORE-MXenes." J.R. and P.E. acknowledge support from the Knut and Alice Wallenberg (KAW) Foundation for Fellowship Grants and Project funding (KAW Grant No. 2015.0043). J.R. also acknowledges funding from Stiftelsen fr Strategisk Forskning (SSF) Program No. EM16-0004.
[1] M. Naguib, M. Kurtoglu, V. Presser, J. Lu, J. Niu, M. Heon, L. Hultman, Y. Gogotsi, and M. W. Barsoum, Adv. Mater. 23, 4248 (2011).

[2] M. Naguib, O. Mashtalir, J. Carle, V. Presser, J. Lu, L. Hultman, Y. Gogotsi, and M. W. Barsoum, ACS Nano 6, 1322 (2012).

[3] M. W. Barsoum, MAX Phases: Properties of Machinable Ternary Carbides and Nitrides (Wiley, New York, 2013).

[4] C. Hu, C.-C. Lai, Q. Tao, J. Lu, J. Halim, L. Sun, J. Zhang, J. Yang, B. Anasori, J. Wang, Y. Sakka, L. Hultman, P. Eklund, J. Rosen, and M. W. Barsoum, Chem. Commun. (UK) 51, 6560 (2015).

[5] M. Naguib, V. N. Mochalin, M. W. Barsoum, and Y. Gogotsi, Adv. Mater. 26, 992 (2014).

[6] M. Ghidiu, M. R. Lukatskaya, M.-Q. Zhao, Y. Gogotsi, and M. W. Barsoum, Nature (London) 516, 78 (2014).

[7] J. Halim, K. M. Cook, M. Naguib, P. Eklund, Y. Gogotsi, J. Rosen, and M. W. Barsoum, Appl. Surf. Sci. 362, 406 (2016).

[8] A. Miranda, J. Halim, M. Barsoum, and A. Lorke, Appl. Phys. Lett. 108, 033102 (2016).

[9] Y. Dall'Agnese, M. R. Lukatskaya, K. M. Cook, P.-L. Taberna, Y. Gogotsi, and P. Simon, Electrochem. Commun. 48, 118 (2014).

[10] B. Anasori, Y. Xie, M. Beidaghi, J. Lu, B. C. Hosler, L. Hultman, P. R. Kent, Y. Gogotsi, and M. W. Barsoum, ACS Nano 9, 9507 (2015).
[11] M. Khazaei, A. Ranjbar, M. Arai, T. Sasaki, and S. Yunoki, J. Mater. Chem. C 5, 2488 (2017).

[12] A. N. Enyashin and A. L. Ivanovskii, Comput. Theor. Chem. 989, 27 (2012).

[13] A. Enyashin and A. Ivanovskii, J. Solid State Chem. 207, 42 (2013).

[14] M. R. Lukatskaya, O. Mashtalir, C. E. Ren, Y. Dall' Agnese, P. Rozier, P. L. Taberna, M. Naguib, P. Simon, M. W. Barsoum, and Y. Gogotsi, Science 341, 1502 (2013).

[15] S. Lee, S. Yu, F. Shahzad, W. N. Kim, C. Park, S. M. Hong, and C. M. Koo, Nanoscale 9, 13432 (2017).

[16] M. Han, X. Yin, H. Wu, Z. Hou, C. Song, X. Li, L. Zhang, and L. Cheng, ACS Appl. Mater. Interfaces 8, 21011 (2016).

[17] B. Xu, M. Zhu, W. Zhang, X. Zhen, Z. Pei, Q. Xue, C. Zhi, and P. Shi, Adv. Mater. 28, 3333 (2016).

[18] Y. Ma, N. Liu, L. Li, X. Hu, Z. Zou, J. Wang, S. Luo, and Y. Gao, Nat. Commun. 8, 1207 (2017).

[19] H. Liu, C. Duan, C. Yang, W. Shen, F. Wang, and Z. Zhu, Sens. Actuators B: Chem. 218, 60 (2015).

[20] F. M. Römer, U. Wiedwald, T. Strusch, J. Halim, E. Mayerberger, M. W. Barsoum, and M. Farle, RSC Adv. 7, 13097 (2017).

[21] M. Naguib, J. Halim, J. Lu, K. M. Cook, L. Hultman, Y. Gogotsi, and M. W. Barsoum, J. Am. Chem. Soc. 135, 15966 (2013). 
[22] X. Xie, S. Chen, W. Ding, Y. Nie, and Z. Wei, Chem. Commun. (UK) 49, 10112 (2013).

[23] Q. Hu, D. Sun, Q. Wu, H. Wang, L. Wang, B. Liu, A. Zhou, and J. He, J. Phys. Chem. A 117, 14253 (2013).

[24] H. Kim, B. Anasori, Y. Gogotsi, and H. N. Alshareef, Chem. Mater. 29, 6472 (2017).

[25] G. Ying, A. D. Dillon, A. T. Fafarman, and M. W. Barsoum, Materials Research Letters, 5, 391 (2017).

[26] J. Halim, M. R. Lukatskaya, K. M. Cook, J. Lu, C. R. Smith, L.-Å. Näslund, S. J. May, L. Hultman, Y. Gogotsi, P. Eklund, and M. W. Barsoum, Chem. Mater. 26, 2374 (2014).

[27] M. R. Lukatskaya, S. Kota, Z. Lin, M.-Q. Zhao, N. Shpigel, M. D. Levi, J. Halim, P.-L. Taberna, M. W. Barsoum, P. Simon, and Y. Gogotsi, Nat. Energy 2, 17105 (2017).

[28] Q. Tao, M. Dahlqvist, J. Lu, S. Kota, R. Meshkian, J. Halim, J. Palisaitis, L. Hultman, M. W. Barsoum, P. O. Å Persson, and J. Rosen, Nat. Commun. 8, 14949 (2017).

[29] L. Qin, Q. Tao, A. El Ghazaly, J. Fernandez-Rodriguez, P. O. Persson, J. Rosen, and F. Zhang, Adv. Funct. Mater. 28, 1703808 (2018).

[30] Z. W. Seh, K. D. Fredrickson, B. Anasori, J. Kibsgaard, A. L. Strickler, M. R. Lukatskaya, Y. Gogotsi, T. F. Jaramillo, and A. Vojvodic, ACS Energy Lett. 1, 589 (2016).

[31] P. Srimuk, J. Halim, J. Lee, Q. Tao, J. Rosen, and V. Presser, ACS Sustainable Chem. Eng. 6, 3739 (2018).

[32] J. Halim, S. Kota, M. R. Lukatskaya, M. Naguib, M.-Q. Zhao, E. J. Moon, J. Pitock, J. Nanda, S. J. May, Y. Gogotsi, and M. W. Barsoum, Adv. Funct. Mater. 26, 3118 (2016).

[33] B. Anasori, J. Halim, J. Lu, C. A. Voigt, L. Hultman, and M. W. Barsoum, Scr. Mater. 101, 5 (2015).

[34] B. Anasori, M. Dahlqvist, J. Halim, E. J. Moon, J. Lu, B. C. Hosler, E. N. Caspi, S. J. May, L. Hultman, P. Eklund, J. Rosén, and M. W. Barsoum, J. Appl. Phys. 118, 094304 (2015).

[35] See Supplemental Material at http://link.aps.org/supplemental/ 10.1103/PhysRevB.98.104202 for details on SEM micrographs and XRD patterns of the MXene samples, and transport and Hall data.

[36] A. Lipatov, M. Alhabeb, M. R. Lukatskaya, A. Boson, Y. Gogotsi, and A. Sinitskii, Adv. Electron. Mater. 2, 1600255 (2016).

[37] B. Anasori, C. Shi, E. J. Moon, Y. Xie, C. A. Voigt, P. R. Kent, S. J. May, S. J. Billinge, M. W. Barsoum, and Y. Gogotsi, Nanoscale Horiz. 1, 227 (2016).

[38] P. Collini, S. Kota, A. D. Dillon, M. W. Barsoum, and A. T. Fafarman, J. Electrochem. Soc. 164, D573 (2017).

[39] C. J. Zhang, S. Pinilla, N. McEvoy, C. P. Cullen, B. Anasori, E. Long, S.-H. Park, A. Seral-Ascaso, A. Shmeliov, D. Krishnan, C. Morant, X. Liu, G. S. Duesberg, Y. Gogotsi, and V. Nicolosi, Adv. Mater. 29, 4848 (2017).

[40] H. Lind, J. Halim, S. I. Simak, and J. Rosen, Phys. Rev. Mater. 1, 044002 (2017).

[41] C. Adkins, J. Phys.: Condens. Matter 1, 1253 (1989).

[42] T. Chui, G. Deutscher, P. Lindenfeld, and W. L. McLean, Phys Rev B 23, 6172(R) (1981).

[43] C. Grimaldi, P. Ryser, and S. Strässler, J. Appl. Phys. 88, 4164 (2000).

[44] P. Guyot-Sionnest, J. Phys. Chem. Lett. 3, 1169 (2012).
[45] O. Entin-Wohlman, Y. Gefen, and Y. Shapira, J. Phys. C: Solid State Phys. 16, 1161 (1983).

[46] P. Sheng and J. Klafter, Phys. Rev. B 27, 2583 (1983).

[47] M. P. J. van Staveren, H. Brom, and L. De Jongh, Phys. Rep. 208, 1 (1991).

[48] Y. Yi, Acta Mater. 56, 2810 (2008).

[49] M. Affronte, M. Campani, S. Piccinini, M. Tamborin, B. Morten, M. Prudenziati, and O. Laborde, J. Low Temp. Phys. 109, 461 (1997).

[50] I. S. Beloborodov, A. V. Lopatin, and V. M. Vinokur, Phys. Rev. B 72, 125121 (2005).

[51] T. Scabarozi, A. Ganguly, J. Hettinger, S. Lofland, S. Amini, P. Finkel, T. El-Raghy, and M. Barsoum, J. Appl. Phys. 104, 073713 (2008).

[52] M. Naguib, R. R. Unocic, B. L. Armstrong, and J. Nanda, Dalton Trans. 44, 9353 (2015).

[53] N. F. Mott and E. A. Davis, Electronic Processes in NonCrystalline Materials (Oxford University, New York, 2012).

[54] M. Pollak, J. Non-Cryst. Solids 8, 486 (1972).

[55] A. Efros and B. Shklovskii, J. Phys. C 8, L49 (1975).

[56] A. Möbius, C. Frenzel, R. Thielsch, R. Rosenbaum, C. J. Adkins, M. Schreiber, H.-D. Bauer, R. Grötzschel, V. Hoffmann, T. Krieg, N. Matz, H. Vinzelberg, and M. Witcomb, Phys. Rev. B 60, 14209 (1999).

[57] P. W. Anderson, in A Career In Theoretical Physics (World Scientific, Singapore, 2004), p. 537.

[58] C. Godet, J. Non-Cryst. Solids 299, 333 (2002).

[59] C. Godet, Phys. Status Solidi B 231, 499 (2002).

[60] H. Qiu, T. Xu, Z. Wang, W. Ren, H. Nan, Z. Ni, Q. Chen, S. Yuan, F. Miao, and F. Song, Nat. Commun. 4, 2642 (2013).

[61] K. Abkemeier, C. Adkins, R. Asal, and E. Davis, J. Phys.: Condens. Matter 4, 9113 (1992).

[62] O. Gunnarsson, M. Calandra, and J. Han, Rev. Mod. Phys. 75, 1085 (2003).

[63] T. Ouisse, Phys. Status Solidi A 162, 339 (1997).

[64] I. Balberg, J. Phys. D 42, 064003 (2009).

[65] A. Melzi and A. Chiquito, J. Phys. D 49, 315303 (2016).

[66] I. Austin and N. F. Mott, Adv. Phys. 18, 41 (1969).

[67] T. Ouisse and M. W. Barsoum, Mater. Res. Lett. 5, 365 (2017).

[68] G. Bergmann, Phys. Rep. 107, 1 (1984).

[69] A. El Kaaouachi, A. Nafidi, A. Nafidi, and G. Biskupski, Semicond. Sci. Technol. 18, 69 (2002).

[70] V. L. Nguen, B. Z. Spivak, and B. I. Shklovskii, Zh. Eksp. Teor. Fiz. 89, 1770 (1985) [JETP 62, 1021 (1985)].

[71] F. Tremblay, M. Pepper, D. Ritchie, D. C. Peacock, J. E. F. Frost, and G. A. C. Jones, Phys. Rev. B 39, 8059(R) (1989).

[72] B. Capoen and G. Biskupski, Phys. Scr. 65, 119 (2002).

[73] I. Persson, L.-Å. Näslund, J. Halim, M. W. Barsoum, V. Darakchieva, J. Palisaitis, J. Rosen, and P. O. A. Persson, 2D Mater. 5, 015002 (2017).

[74] M. Ghidiu, S. Kota, J. Halim, A. W. Sherwood, N. Nedfors, J. Rosen, V. N. Mochalin, and M. W. Barsoum, Chem. Mater. 29, 1099 (2017).

[75] K. D. Fredrickson, B. Anasori, Z. W. Seh, Y. Gogotsi, and A. Vojvodic, J. Phys. Chem. C 120, 28432 (2016).

[76] M. Ghidiu, J. Halim, S. Kota, D. Bish, Y. Gogotsi, and M. W. Barsoum, Chem. Mater. 28, 3507 (2016). 